\title{
Impinging Howarth stagnation-point flows
}

\author{
M. R. Turner \\ Department of Mathematics \\ University of Surrey \\ Guildford, Surrey GU2 7XH \\ United Kingdom \\ P. D. Weidman \\ Department of Mechanical Engineering \\ University of Colorado \\ Boulder, CO 80309-0427 USA
}

\begin{abstract}
The flow of one Howarth stagnation-point flow impinging directly on another Howarth stagnation-point flow is studied, and an exact similarity solution to the Navier-Stokes equations is found. The upper layer fluid has density $\rho_{1}$ and kinematic viscosity $\nu_{1}$ while the lower layer fluid has density $\rho_{2}$ and kinematic viscosity $\nu_{2}$ and the two fluids are assumed to be immiscible. This problem has potentially five independent parameters to investigate, but application of the continuity of the normal stresses at the interface imposes restrictions which reduces the problem to one with three independent parameters, namely a ratio $\sigma$ of strain rates and the fluid parameter ratios $\rho=\rho_{1} / \rho_{2}$ and $\nu=\nu_{1} / \nu_{2}$. Numerical results are presented for selected values of $\rho$ and $\nu$ for a range of $\sigma$ and show that stable results exist for all values of $\sigma>0$, and for a range of negative $\sigma$ values. Sample stable velocity profiles are also presented.
\end{abstract}

\section{Introduction}

Impinging stagnation-point flows have been a matter of interest over the years. Wang (1987) was the first to investigate impinging Hiemenz (1911) and Homann (1936) stagnation-point flows and subsequently found exact solutions to these problems in the form of a similarity solution. Hiemenz flow is a planar stagnation-point flow while Homann flow is an axisymmetric stagnation-point flow both of which imping on a fixed flat plate. Tilley and Weidman (1998) extended the similarity solution idea to obtain solutions to the problem of impinging oblique planar stagnation-point flows and Weidman (2017) reported results for normally impinging axisymmetric rotational stagnation-point flows.

These studies led us to consider new possible impinging stagnation-point flow problems. In the first, one Hiemenz stagnation flow impinges on another Hiemenz stagnation flow 
oriented orthogonal to the other. Here one finds that no similarity solution exists unless the strain rates of the two flows are equal. Another possibility is the impingement of a Hiemenz stagnation flow on a Homann stagnation flow, but here again no similarity solution exists because the different geometries of the two problems lead to equations with no solutions. Then one could envision an axisymmetric rotational stagnation-point flow impinging on a Homann stagnation-point flow, but again no similarity solution is available again due to the geometries of the two problems. Consequently, it appears that the only remaining possibility for study is the normal impingement of two Howarth (1951) stagnation-point flows which is the subject of this investigation. The Howarth (1951) flow is one composed of two orthogonal stagnation-point flows with different strain rates in the two directions impinging on a fixed flat plate. Such impinging stagnation-point flows occur in transpiration cooling, and injection cooling problems (Wang, 1964).

The presentation of the paper is as follows. In $\S 2$ we derive the similarity equations governing the flow and show that restrictions on continuity of the normal stress at the interface reduces the problem to three independent parameters, namely a strain rate ratio $\sigma$ and the fluid parameters $\rho=\rho_{1} / \rho_{2}$ and $\nu=\nu_{1} / \nu_{2}$. Numerical solutions for fixed values of the fluid parameters over a range of $\sigma$ are presented in $\S 3$. The stability of the dual solutions encountered are analyzed in $\S 4$, with sample velocity profiles along the stable branches presented in $\S 5$. The paper ends with a discussion of results and concluding remarks in $\S 6$.

\section{Problem formulation}

We consider a steady Howarth stagnation-point flow with strain rates $(a, b)$, density $\rho_{1}$ and kinematic viscosity $\nu_{1}$ in the upper layer impinging on another steady Howarth stagnationpoint flow of strain rates $(c, d)$ of density $\rho_{2}$ and kinematic viscosity $\nu_{2}$ in the lower layer along the plane $z=0$, see the schematic of this setup in figure 1 . Both fluids are assumed to be incompressible and immiscible, and the fluid interface is assumed to remain at $z=0$. Using Cartesian coordinates $(x, y, z)$ with corresponding velocities $(u, v, w)$, we follow Howarth (1951) and take the upper layer velocities $(z>0)$ as

$$
u_{1}=a x f_{1}^{\prime}(\eta), \quad v_{1}=b y g_{1}^{\prime}(\eta), \quad \eta=\sqrt{\frac{a}{\nu_{1}}} z
$$

where we have posited the Howarth stagnation-point flow in the far field as $u_{1}=a x$ and $v_{1}=b y$, and $\eta$ is a dimensionless variable. The equation of mass continuity then requires that the velocity normal to the stagnation plane be

$$
w_{1}=-\sqrt{a \nu_{1}}\left[f_{1}(\eta)+\sigma_{1} g_{1}(\eta)\right]
$$


where $\sigma_{1}=b / a$ is the ratio of strain rate along the $y$ coordinate to that along the $x$ coordinate. Inserting this ansatz into the Navier-Stokes equations gives

$$
\begin{gathered}
-\frac{1}{\rho_{1}} \frac{\partial p_{1}}{\partial x}=a^{2} x\left[f_{1}^{\prime 2}-\left(f_{1}+\sigma_{1} g_{1}\right) f_{1}^{\prime \prime}-f_{1}^{\prime \prime \prime}\right] \\
-\frac{1}{\rho_{1}} \frac{\partial p_{1}}{\partial y}=b^{2} y\left[g_{1}^{\prime 2}-\left(\frac{1}{\sigma_{1}} f_{1}+g_{1}\right) g_{1}^{\prime \prime}-\frac{1}{\sigma_{1}} g_{1}^{\prime \prime \prime}\right] \\
-\frac{1}{\rho_{1}} \frac{\partial p_{1}}{\partial \eta}=\frac{\nu_{1}}{a}\left(a f_{1}+b g_{1}\right)\left(a f_{1}^{\prime}+b g_{1}^{\prime}\right)+\nu_{1}\left(a f_{1}^{\prime \prime}+b g_{1}^{\prime \prime}\right) .
\end{gathered}
$$

Observing that the latter equation depends on $\eta$ only, we obtain from (2.3a) and (2.3b) the coupled set of ODEs

$$
\begin{gathered}
f_{1}^{\prime \prime \prime}+\left(f_{1}+\sigma_{1} g_{1}\right) f_{1}^{\prime \prime}-f_{1}^{\prime 2}+1=0 \\
g_{1}^{\prime \prime \prime}+\left(f_{1}+\sigma_{1} g_{1}\right) g_{1}^{\prime \prime}-\sigma_{1} g_{1}^{\prime 2}+\sigma_{1}=0
\end{gathered}
$$

where the constants are found by applying the far-field conditions $f_{1}^{\prime}(\infty)=g_{1}^{\prime}(\infty)=1$. Next, computation of the pressure in the upper layer gives

$$
p_{1}=p_{10}-\rho_{1}\left(\frac{a^{2} x^{2}}{2}+\frac{b^{2} y^{2}}{2}\right)-a \rho_{1} \nu_{1}\left[\frac{\left(f_{1}+\sigma_{1} g_{1}\right)^{2}}{2}+\left(f_{1}^{\prime}+\sigma_{1} g_{1}^{\prime}\right)\right]
$$

where $p_{10}$ is the constant pressure at $(0,0,0)$.

In the lower layer $(z<0)$ we take $z$ pointing downwards and posit the Howarth stagnationpoint flow in the far field as $u_{2}=c x$ and $v_{2}=d y$. Then we choose the similarity formulation as

$$
u_{2}=c x f_{2}^{\prime}(\zeta), \quad v_{2}=d y g_{2}^{\prime}(\zeta), \quad \zeta=\sqrt{\frac{c}{\nu_{2}}} z
$$

which satisfies the continuity equation when

$$
w_{2}=-\sqrt{c \nu_{2}}\left[f_{2}(\zeta)+\sigma_{2} g_{2}(\zeta)\right]
$$

where $\sigma_{2}=d / c$. Following the upper layer analysis one readily finds the governing coupled ODEs

$$
\begin{gathered}
f_{2}^{\prime \prime \prime}+\left(f_{2}+\sigma_{2} g_{2}\right) f_{2}^{\prime \prime}-f_{2}^{\prime 2}+1=0 \\
g_{2}^{\prime \prime \prime}+\left(f_{2}+\sigma_{2} g_{2}\right) g_{2}^{\prime \prime}-\sigma_{2} g_{2}^{\prime 2}+\sigma_{2}=0
\end{gathered}
$$

and the lower layer pressure field is

$$
p_{2}=p_{20}-\rho_{2}\left(\frac{c^{2} x^{2}}{2}+\frac{d^{2} y^{2}}{2}\right)-c \rho_{2} \nu_{2}\left[\frac{\left(f_{2}+\sigma_{2} g_{2}\right)^{2}}{2}+\left(f_{2}^{\prime}+\sigma_{2} g_{2}^{\prime}\right)\right]
$$


with $p_{2}=p_{20}$ at $(0,0,0)$.

We now turn to the conditions to be met at the interface $z=0$. First there is no normal velocity through the interface and hence

$$
f_{1}(0)=g_{1}(0)=f_{2}(0)=g_{2}(0)=0
$$

and for equal velocities at the interface we have

$$
a f_{1}^{\prime}(0)=c f_{2}^{\prime}(0) ; \quad b g_{1}^{\prime}(0)=d g_{2}^{\prime}(0)
$$

The normal stresses $-p+2 \mu w_{z}$ must be equal at the interface which gives

$$
\begin{aligned}
& p_{10}-\rho_{1}\left(\frac{a^{2} x^{2}}{2}+\frac{b^{2} y^{2}}{2}\right)-a \rho_{1} \nu_{1}\left(f_{1}^{\prime}(0)+\sigma_{1} g_{1}^{\prime}(0)\right)+2 a \mu_{1}\left(f_{1}^{\prime}(0)+\sigma_{1} g_{1}^{\prime}(0)\right)= \\
& p_{20}-\rho_{2}\left(\frac{c^{2} x^{2}}{2}+\frac{d^{2} y^{2}}{2}\right)-c \rho_{2} \nu_{2}\left(f_{2}^{\prime}(0)+\sigma_{2} g_{2}^{\prime}(0)\right)+2 c \mu_{2}\left(f_{2}^{\prime}(0)+\sigma_{2} g_{2}^{\prime}(0)\right)
\end{aligned}
$$

where use has been made of Eq. (2.10). Equating on $x^{2}$ and $y^{2}$ requires

$$
c=\rho^{1 / 2} a, \quad d=\rho^{1 / 2} b, \quad \text { where } \quad \rho=\frac{\rho_{1}}{\rho_{2}} .
$$

Thus we find $\sigma_{2}=d / c=b / a=\sigma_{1} \equiv \sigma$. Note that $\sigma>0$ indicates that both strains at $z=\infty$ (similarly at $z=-\infty)$ are directed away from the line $x=y=0$, while for $\sigma<0$ the strain at $z=\infty$ in the $x$-direction, say, is directed away from this line while the strain in the $y$-direction is directed towards this line. This then affects the down-flow velocity in each half-space, see (2.2) and (2.7). Using this definition for $\sigma$ the interfacial conditions (2.11) may be written

$$
f_{1}^{\prime}(0)=\rho^{1 / 2} f_{2}^{\prime}(0) ; \quad g_{1}^{\prime}(0)=\rho^{1 / 2} g_{2}^{\prime}(0)
$$

and inserting this into the remainder of $(2.12)$ gives the relation between the constants $p_{10}$ and $p_{20}$, viz.

$$
p_{20}=p_{10}+a \rho^{1 / 2}\left(\mu_{1}-\mu_{2}\right)\left[f_{2}^{\prime}(0)+\sigma g_{2}^{\prime}(0)\right] .
$$

Finally we must have equal horizontal interface stresses which leads to the requirements

$$
f_{2}^{\prime \prime}(0)=-\rho^{1 / 4} \nu^{1 / 2} f_{1}^{\prime \prime}(0) ; \quad g_{2}^{\prime \prime}(0)=-\rho^{1 / 4} \nu^{1 / 2} g_{1}^{\prime \prime}(0)
$$

where $\nu=\nu_{1} / \nu_{2}$.

The equations and boundary conditions are now summarized making use of the fact that $\sigma_{2}=\sigma_{1} \equiv \sigma$. The governing equations in each layer

$$
f_{1}^{\prime \prime \prime}+\left(f_{1}+\sigma g_{1}\right) f_{1}^{\prime \prime}-f_{1}^{\prime 2}+1=0
$$




$$
\begin{gathered}
g_{1}^{\prime \prime \prime}+\left(f_{1}+\sigma g_{1}\right) g_{1}^{\prime \prime}-\sigma g_{1}^{\prime 2}+\sigma=0 \\
f_{2}^{\prime \prime \prime}+\left(f_{2}+\sigma g_{2}\right) f_{2}^{\prime \prime}-f_{2}^{\prime 2}+1=0 \\
g_{2}^{\prime \prime \prime}+\left(f_{2}+\sigma g_{2}\right) g_{2}^{\prime \prime}-\sigma g_{2}^{\prime 2}+\sigma=0
\end{gathered}
$$

are to be solved with interfacial conditions (2.10), (2.14) and (2.16) along with the far-field conditions

$$
f_{1}^{\prime}(\infty)=f_{2}^{\prime}(\infty)=g_{1}^{\prime}(\infty)=g_{2}^{\prime}(\infty)=1
$$

Potentially this problem could have been governed by five parameters, namely the three strain rate ratios $\sigma, c / a, d / a$ and the fluid parameters $\rho$ and $\nu$. But owing to the conditions wrought by equality of normal stress at the interface the problem depends only on $\sigma, \rho$ and $\nu$.

\subsection{Reduction to impinging Hiemenz and Homann flows}

The problems considered by Wang (1987) may be recovered from the general case as follows. First, upon setting $a=b$ which gives $\sigma=1$ we note that the equations (2.17) are satisfied by setting $g_{1}=f_{1}$ and $g_{2}=f_{2}$ which furnish the equations

$$
\begin{aligned}
& f_{1}^{\prime \prime \prime}+2 f_{1} f_{1}^{\prime \prime}-f_{1}^{\prime 2}+1=0 \\
& f_{2}^{\prime \prime \prime}+2 f_{2} f_{2}^{\prime \prime}-f_{2}^{\prime 2}+1=0
\end{aligned}
$$

to be solved with interfacial conditions

$$
f_{1}(0)=f_{2}(0)=0, \quad f_{1}^{\prime}(0)=\rho^{1 / 2} f_{2}^{\prime}(0), \quad f_{2}^{\prime \prime}(0)=-\rho^{1 / 4} \nu^{1 / 2} f_{1}^{\prime \prime}(0)
$$

and far-field conditions

$$
f_{1}^{\prime}(\infty)=f_{2}^{\prime}(\infty)=1
$$

These are recognized as the impinging Homann stagnation flows of Wang (1957). Similarly, setting $b=d=0$ giving $\sigma=0$ reduces the impinging Howarth stagnation flows to impinging Hiemenz stagnation flows. However, we note that the pressure relation given by Eq. (7) of Wang (1957) is valid only for equality of pressure at the interface and not equality of normal stress. Fortunately this does not alter the governing equations. Concerning the boundary conditions, we see that the interfacial stress relation given in Eq. (16) of Wang (1957) has a typographical error; the subscripts on $f^{\prime \prime}(0)$ should be interchanged. 


\section{Numerical results}

The coupled set of ODEs (2.17) along with the boundary conditions (2.18) are solved numerically using a shooting technique. The ODEs are integrated from $\eta=0$ to $\eta=\eta_{\max }$ via $4^{\text {th }}$ order Runge-Kutta integration and Newton iterations are used to update the initial guesses for the unknown interface quantities $f_{1}^{\prime}(0), g_{1}^{\prime}(0), f_{1}^{\prime \prime}(0)$ and $g_{1}^{\prime \prime}(0)$ using equations (2.18d), see Press, et al (1989). The domain of integration $\eta \in\left[0, \eta_{\max }\right]$ and the Runge-Kutta step size were varied to ensure the results presented in this paper have converged. For $\sigma \gtrsim-3 / 4$ a value of $\eta_{\max }=10$ is suitable for convergence, but for smaller $\sigma$ values this needs to be increased to $\eta_{\max }=40$ due to a rapid thickening of the solution boundary layer, as will be shown in the following results.

Sample solutions are now provided at selected values of $\rho$ and $\nu$. We begin with $\nu=1.0$ varying the density ratio $\rho=\{0.2,0.4,0.6,0.8\}$. The results for the interfacial velocities $f_{1}^{\prime}(0)$ and $g_{1}^{\prime}(0)$ and the interfacial shear stresses $f_{1}^{\prime \prime}(0)$ and $g_{1}^{\prime \prime}(0)$ are presented in figures 2a,b,c,d. Of particular note is the appearance of a unique solution for $\sigma>0$, dual solutions for $\sigma_{1}<\sigma<0$, a unique solution at $\sigma=\sigma_{1}$, dual solutions for $\sigma<\sigma_{2}$ and a unique solution at $\sigma=\sigma_{2}$, where the values of $\sigma_{1}$ and $\sigma_{2}$ are listed in Table 1 . Note, these values of $\sigma_{1}$ and $\sigma_{2}$ are different from those introduced in $\S 2$.

\begin{tabular}{|c|c|c|}
\hline$\rho$ & $\sigma_{1}$ & $\sigma_{2}$ \\
\hline \hline 0.2 & -0.28315 & -0.38699 \\
\hline 0.4 & -0.29913 & -0.36983 \\
\hline 0.6 & -0.31255 & -0.35513 \\
\hline 0.8 & -0.32383 & -0.34305 \\
\hline
\end{tabular}

Table 1. Turning points $\sigma_{1}$ and $\sigma_{2}$ for $\nu=1.0$ at the listed values of $\rho$.

A survey was carried out to determine the values of the turning points $\sigma_{1}$ and $\sigma_{2}$ for $\nu=1$ as a function of $\rho$. These results are displayed in figure 3 and careful calculations show that the two curves merge at $\rho=1$ with the value $\sigma_{1}=\sigma_{2}=1 / 3$.

The next set of calculations were carried out for $\rho=0.6$ varying the viscosity ratio $\nu=\{0.1,0.25,4.0,10.0\}$. The results for the interfacial velocities $f_{1}^{\prime}(0)$ and $g_{1}^{\prime}(0)$ and the interfacial shear stresses $f_{1}^{\prime \prime}(0)$ and $g_{1}^{\prime \prime}(0)$ are presented in figures $4 \mathrm{a}, \mathrm{b}, \mathrm{c}, \mathrm{d}$. The same existence of unique and dual solutions appear as for the previous study, where now the values of $\sigma_{1}$ and $\sigma_{2}$ are listed in Table 2 . 


\begin{tabular}{|c|c|c|}
\hline$\nu$ & $\sigma_{1}$ & $\sigma_{2}$ \\
\hline \hline 0.10 & -0.31735 & -0.34980 \\
\hline 0.25 & -0.31508 & -0.35232 \\
\hline 4.0 & -0.31205 & -0.355768 \\
\hline 10.0 & -0.31298 & -0.35483 \\
\hline
\end{tabular}

Table 2. Turning points $\sigma_{1}$ and $\sigma_{2}$ for $\rho=0.6$ at the listed values of $\nu$.

A survey was carried out for the variation of the turning points $\sigma_{1}$ and $\sigma_{2}$ for $\rho=0.6$ as a function of $\nu$. These results are displayed in figure 5 and careful calculations show that the two curves merge at $\nu=0$ with $\sigma_{1}=\sigma_{2}=1 / 3$.

\section{Stability analysis}

By introducing the dimensionless time variable $\tau=$ at, we can now include the unsteady acceleration term in the Navier-Stokes equations, which upon substitution of the similarity solutions (2.1) and (2.6) gives rise to the coupled nonlinear system

$$
\begin{gathered}
f_{1}^{\prime \prime \prime}+\left(f_{1}+\sigma g_{1}\right) f_{1}^{\prime \prime}-f_{1}^{\prime 2}+1-f_{1 \tau}^{\prime}=0 \\
g_{1}^{\prime \prime \prime}+\left(f_{1}+\sigma g_{1}\right) g_{1}^{\prime \prime}-\sigma g_{1}^{\prime 2}+\sigma-g_{1 \tau}^{\prime}=0 \\
f_{2}^{\prime \prime \prime}+\left(f_{2}+\sigma g_{2}\right) f_{2}^{\prime \prime}-f_{2}^{\prime 2}+1-f_{2 \tau}^{\prime}=0 \\
g_{2}^{\prime \prime \prime}+\left(f_{2}+\sigma g_{2}\right) g_{2}^{\prime \prime}-\sigma g_{2}^{\prime 2}+\sigma-g_{2 \tau}^{\prime}=0 .
\end{gathered}
$$

To examine the viscous stability of these self-similar equations, we follow the approaches of Merkin (1985) and Weidman and Turner (2017) and write

$$
\left[f_{1}, g_{1}, f_{2}, g_{2}\right](\eta, t)=\left[f_{10}, g_{10}, f_{20}, g_{20}\right](\eta)+\delta e^{-\lambda \tau}\left[f_{11}, g_{11}, f_{21}, g_{21}\right](\eta)
$$

where $\lambda$ is a complex eigenvalue determining the linear stability, $\delta$ is an amplitude parameter and $\left[f_{10}, g_{10}, f_{20}, g_{20}\right]$ are solutions of the steady problem found in $\S 3$. Linearizing for $\delta \ll 1$ leads to the following coupled set of ODEs for the perturbation quantities

$$
\begin{gathered}
f_{11}^{\prime \prime \prime}+\left(f_{10}+\sigma g_{10}\right) f_{11}^{\prime \prime}+\left(f_{11}+\sigma g_{11}\right) f_{10}^{\prime \prime}-2 f_{10}^{\prime} f_{11}^{\prime}+\lambda f_{11}^{\prime}=0 \\
g_{11}^{\prime \prime \prime}+\left(f_{10}+\sigma g_{10}\right) g_{11}^{\prime \prime}+\left(f_{11}+\sigma g_{11}\right) g_{10}^{\prime \prime}-2 \sigma g_{10}^{\prime} g_{11}^{\prime}+\lambda g_{11}^{\prime}=0 \\
f_{21}^{\prime \prime \prime}+\left(f_{20}+\sigma g_{20}\right) f_{21}^{\prime \prime}+\left(f_{21}+\sigma g_{21}\right) f_{20}^{\prime \prime}-2 f_{20}^{\prime} f_{21}^{\prime}+\lambda f_{21}^{\prime}=0 \\
g_{21}^{\prime \prime \prime}+\left(f_{20}+\sigma g_{20}\right) g_{21}^{\prime \prime}+\left(f_{21}+\sigma g_{21}\right) g_{20}^{\prime \prime}-2 \sigma g_{20}^{\prime} g_{21}^{\prime}+\lambda g_{21}^{\prime}=0 .
\end{gathered}
$$


Solutions to this system are sought with the same boundary conditions as in (2.18) except with the far-field condition (2.18d) replaced with the homogeneous conditions

$$
f_{11}^{\prime}(\infty)=f_{12}^{\prime}(\infty)=g_{11}^{\prime}(\infty)=g_{12}^{\prime}(\infty)=0
$$

The solutions result in an infinite set of eigenvalues with the property $\operatorname{Re}\left(\lambda_{1}\right)<\operatorname{Re}\left(\lambda_{2}\right)<$ $\operatorname{Re}\left(\lambda_{3}\right)<\cdots$ where the real part of $\lambda_{i}$ determines the growth/decay rate of the perturbation. If $\operatorname{Re}\left(\lambda_{1}\right) \geq 0$ then the steady solution is stable.

The system of ODES (4.3) are solved using the same shooting approach as was used for the steady solution, but in this case the homogeneous boundary conditions (4.4) mean we can always multiply our solution by an arbitrary constant. Hence we normalize our result by fixing the interface perturbation velocity in the $y$-direction such that

$$
g_{11}^{\prime}(0)=1
$$

and then solving for the four complex unknowns $f_{11}^{\prime}(0), f_{11}^{\prime \prime}(0), g_{11}^{\prime \prime}(0)$ and $\lambda$.

A detailed analysis was carried out for $\rho=0.8$ and $\nu=1$, but similar calculations for $\rho=0.2,0.4$ and 0.6 show that the eigenvalue structure is almost identical to the $\rho=0.8$ case. The values of the smallest real part of $\lambda_{1}$ on the four branches are displayed in figure 6 . Along branches 1 and $3 \lambda_{1}$ is purely real, however on branch 4 complex values of $\lambda$ exist in the small region $-0.737 \leq \sigma \leq-0.716$, and for $\sigma<-1$, as indicated by the horizontal arrows in the plot. The real part of $\lambda_{1}$ along branch 4 crosses zero at $\sigma \equiv \sigma_{\text {crit }} \approx-1.15$ indicating that the impinging solutions become unstable for $\sigma<\sigma_{\text {crit }}$. An interesting feature of figure 6 is the discontinuity in slope along branch 1 which exists at $\sigma=1.0$. This is precisely the value for impinging Homann stagnation-point flows computed by Wang (1987) and is caused by a second stable eigenvalue $\lambda_{2}$ becoming close to $\lambda_{1}$.

\section{Sample velocity profiles for stable solutions}

Knowing the stability of our solutions we are now in a position to present examples of stable velocity profiles which exist only along all of branch 1 and on branch 4 for $\sigma_{\text {crit }}<\sigma<\sigma_{2}$. We begin with profiles $f^{\prime}(\eta)$ and $g^{\prime}(\eta)$ computed at $\sigma=-0.6$ for the density values $\rho=$ $\{0.2,0.4,0.6,0.8\}$ presented in figures $7 \mathrm{a}$ and $7 \mathrm{~b}$, respectively. Here we see that the edge of the boundary layer in the $x$-direction $\left(f_{1}^{\prime}, f_{2}^{\prime}\right)$ is at $\eta \approx 3$ while for the $y$-direction $\left(g_{1}^{\prime}, g_{2}^{\prime}\right)$ it is twice as thick at $\eta \approx 6$. Reducing $\sigma$ to $\sigma=-1.1$ in figures $8 \mathrm{a}$ and $8 \mathrm{~b}$ shows that the boundary layer thickness for the flows in the $x$-direction is almost unchanged from the $\sigma=-0.6$ result, but that the boundary layer in the $y$-direction has expanded considerably 
(now at $\eta \approx 20$ ). This is due to the reverse flow which now occurs at the interface, $z=0$. It is this rapid thickening of the boundary layer in the $y$-direction which eventually leads to the flow becoming unstable. Figures $9 \mathrm{a}$ and $9 \mathrm{~b}$ exhibit profiles along branch 1 computed at $\sigma=3.0$ for the same density values $\rho=\{0.2,0.4,0.6,0.8\}$, and along this branch we note that the boundary layers thin with increasing $\sigma$, hence the stable nature of the flow for $\sigma>0$.

\section{Discussion and conclusion}

We have investigated the problem of two impinging Howarth stagnation-point flows. By seeking exact results to this problem in the form of similarity solutions, we find the problem is governed by four coupled ODEs which depend upon three independent parameters: $\rho$ the density ratio of the two fluids; $\nu$ the kinematic viscosity ratio; and $\sigma$ the ratio of the flow strain rates at $|z|=\infty$, in the $x$ - and $y$-directions.

Numerical results for various combinations of $\rho, \nu$ and $\sigma$ were presented and in each case the solution structure was as follows: for $\sigma>0$ there exists one stable branch of solutions only; for $\sigma_{1}<\sigma<0$ there exists one stable and one unstable branch of solutions while for $\sigma_{\text {crit }}<\sigma<\sigma_{2}$ there exists one stable and one unstable branch of solutions and for $\sigma<\sigma_{\text {crit }}$ there exists two unstable solution branches. For $\sigma_{2}<\sigma<\sigma_{1}$ there are no similarity solutions to this problem. By examining the velocity profiles for the stable branches, it was noted that the thickness of the boundary layer in the $y$-direction increases as $\sigma$ decreases, and this thickening is accompanied by reverse flow at $z=0$ for $\sigma<-1$. It is this boundary layer thickening which leads to flow instability. 


\section{References}

Hiemenz K., Die Grenzschicht an einem in den gleichförmingen Flussigkeitsstrom eingetauchten geraden Kreiszylinder, Dinglers Poly. J., 326 (1911) 321-410.

Homann, F., Der Einfluss grosser Zähigkeit bei der Strömung um den Zylinder und um die Kugel, Z. angew. Math. Mech. (ZAMM) 16 (1936) 153-164.

Howarth, L., The boundary layer in three dimensional flow. Part II. The flow near a stagnation point, Phil. Mag., 2 (1951) 1433-1440.

Merkin, J. H. On dual solutions occurring in mixed convection in a porous medium, J. Eng. Math., 20 (1985) 171-179.

Press, W. H., Flannery, B. P., S.A. Teukolsky, S. A. and Vetterling W. T., Numerical recipes (Cambridge University Press, Cambridge, 1989).

Tilley, B. and Weidman, P. D., Oblique two-fluid stagnation-point flow, Eur. J. Mech. B/Fluids, 17 (1998) 205-217.

Wang, C. Y., Contours for stagnation-point mass injection in hypersonic flow. AIAA Journal, 2(1) (1964) 178-179.

Wang, C. Y., Impinging stagnation flows, Phys. Fluids, 30 (1987) 915-917.

Weidman, P. D., Impinging rotational stagnation-point flows, International Journal of NonLinear Mechanics, 88 (2017) 97-101.

Weidman, P. D. and Turner, M. R., Stagnation-point flows with stretching surfaces: A unified formulation and new results. Euro. J. Mech. B/Fluids 61 (2017) 144-153. 


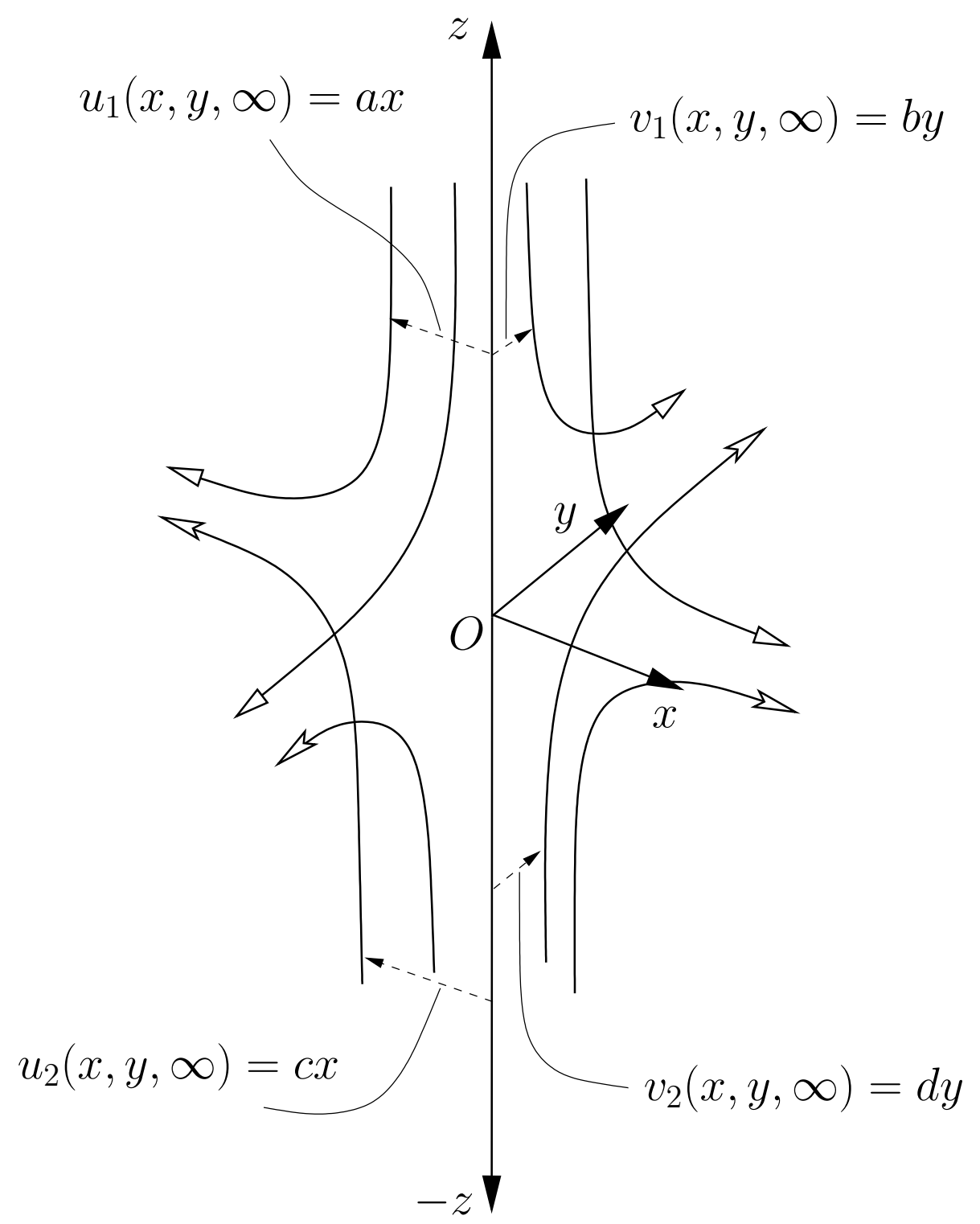

Figure 1. Schematic diagram for impinging Howarth stagnation point flows along the plane $z=0$. 

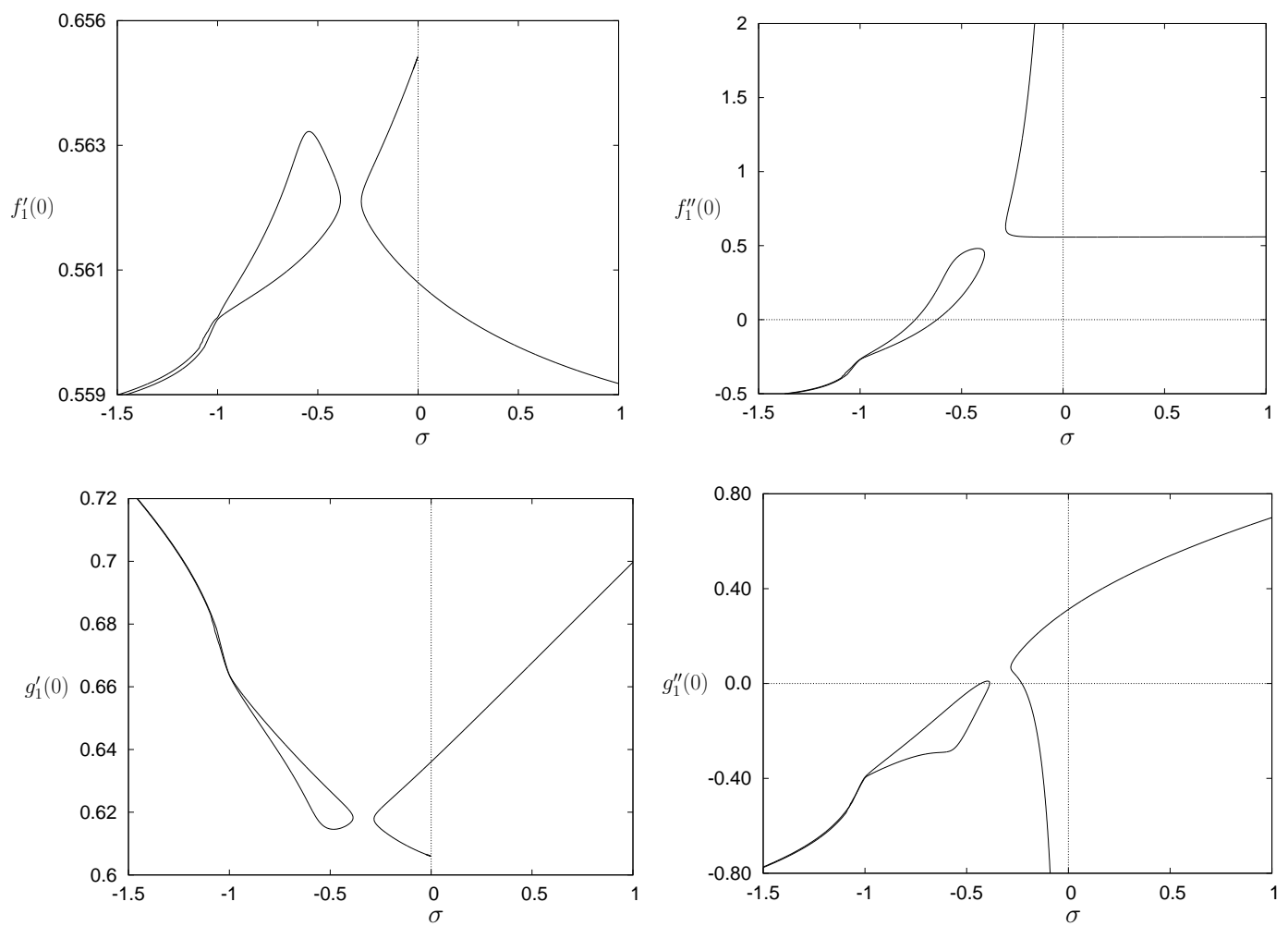

Figure 2a. Interfacial velocities $f_{1}^{\prime}(0), g_{1}^{\prime}(0)$, and upper layer shear stress parameters $f_{1}^{\prime \prime}(0), g_{1}^{\prime \prime}(0)$ for $\rho=0.2$ and $\nu=1.0$. The gap in the solutions lies in the region $\sigma_{2}<\sigma<\sigma_{1}$, where $\sigma_{1}=-0.2832$ and $\sigma_{2}=-0.3870$. 

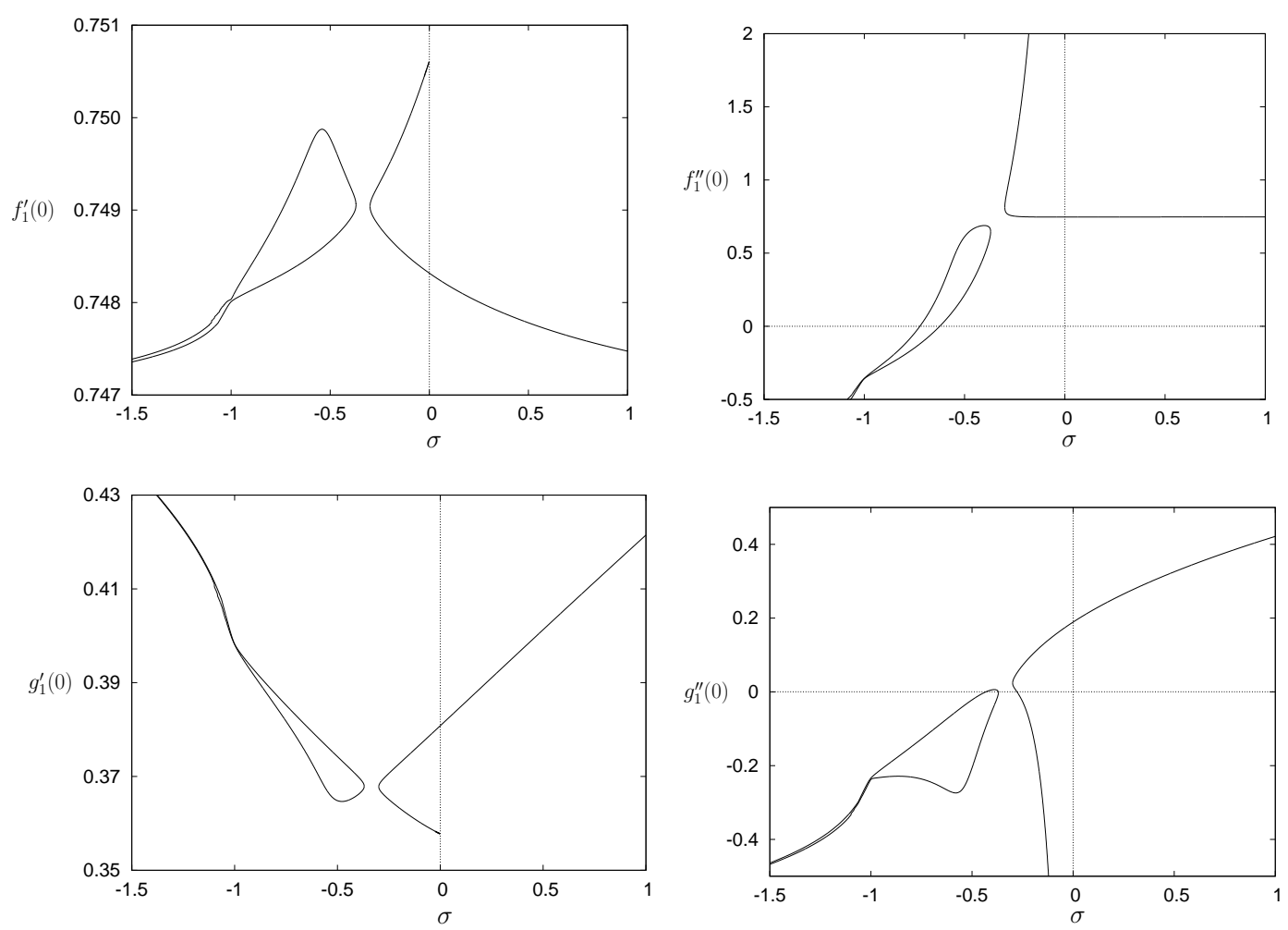

Figure 2b. Interfacial velocities $f_{1}^{\prime}(0), g_{1}^{\prime}(0)$, and upper layer shear stress parameters $f_{1}^{\prime \prime}(0), g_{1}^{\prime \prime}(0)$ for $\rho=0.4$ and $\nu=1.0$. The gap in the solutions lies in the region $\sigma_{2}<\sigma<\sigma_{1}$, where $\sigma_{1}=-0.2991$ and $\sigma_{2}=-0.3698$. 

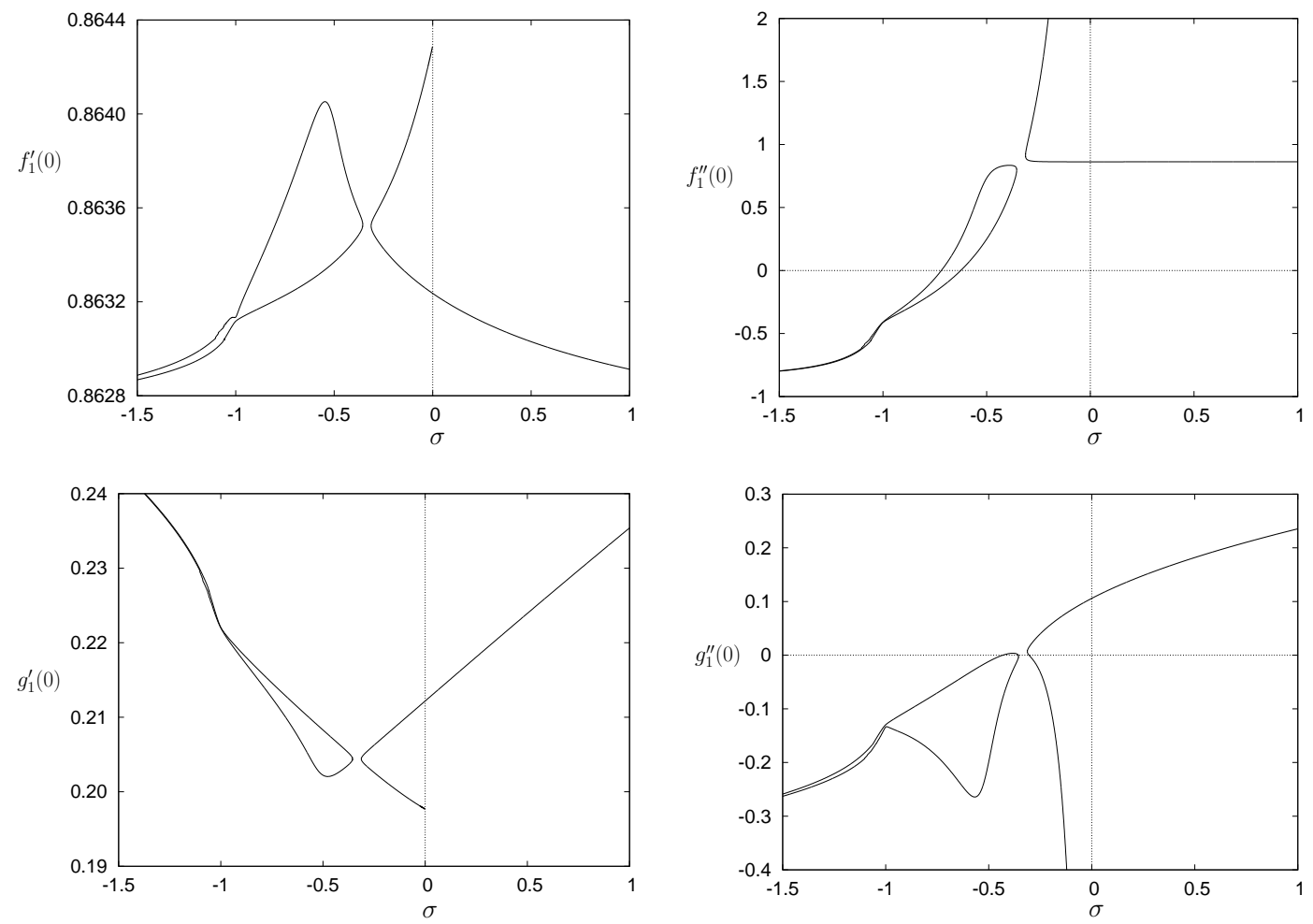

Figure 2c. Interfacial velocities $f_{1}^{\prime}(0), g_{1}^{\prime}(0)$, and upper layer shear stress parameters $f_{1}^{\prime \prime}(0), g_{1}^{\prime \prime}(0)$ for $\rho=0.6$ and $\nu=1.0$. The gap in the solutions lies in the region $\sigma_{2}<\sigma<\sigma_{1}$, where $\sigma_{1}=-0.3126$ and $\sigma_{2}=-0.3551$. 

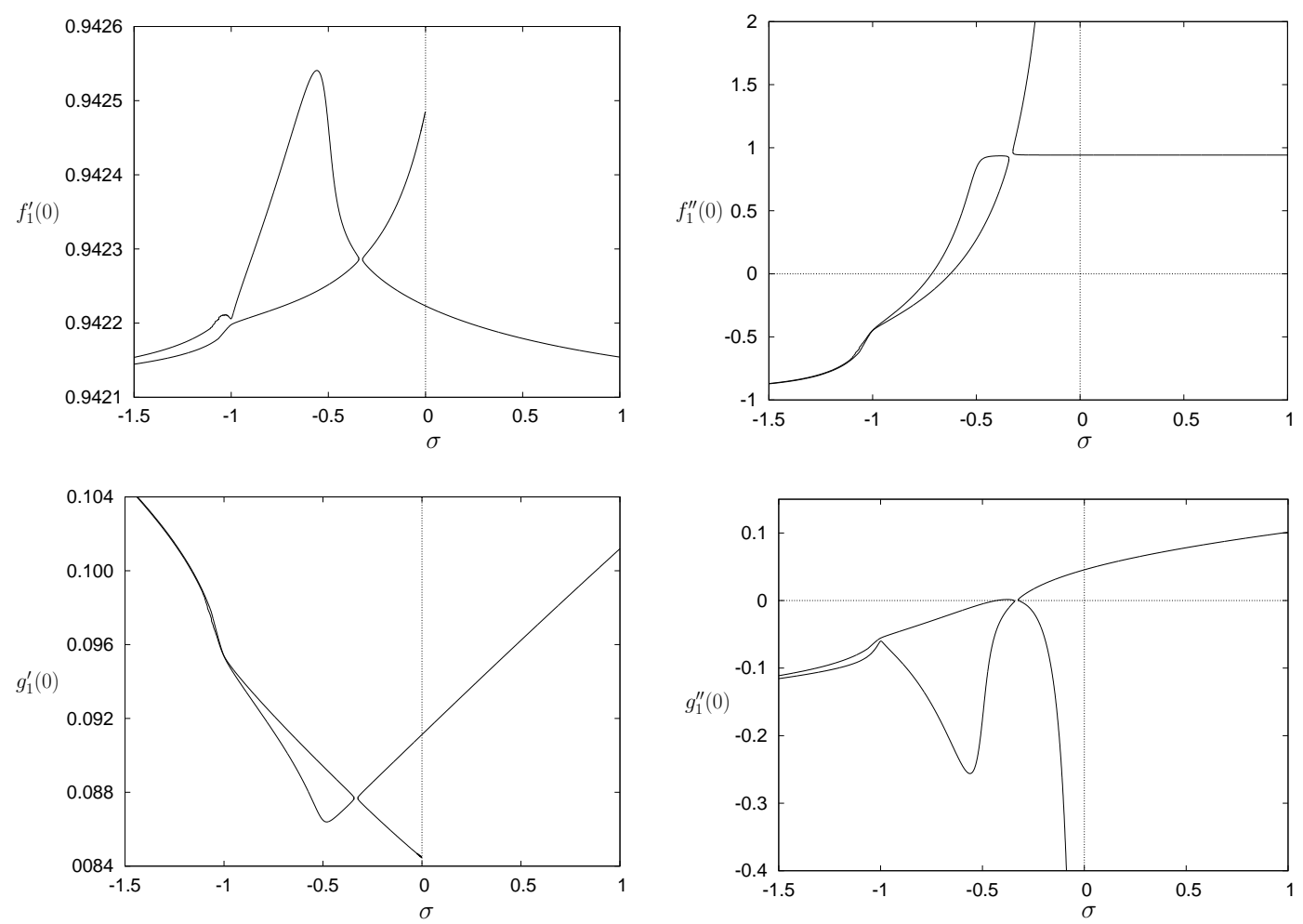

Figure 2d. Interfacial velocities $f_{1}^{\prime}(0), g_{1}^{\prime}(0)$, and upper layer shear stress parameters $f_{1}^{\prime \prime}(0), g_{1}^{\prime \prime}(0)$ for $\rho=0.8$ and $\nu=1.0$. The gap in the solutions lies in the region $\sigma_{2}<\sigma<\sigma_{1}$, where $\sigma_{1}=-0.3238$ and $\sigma_{2}=-0.3431$. 


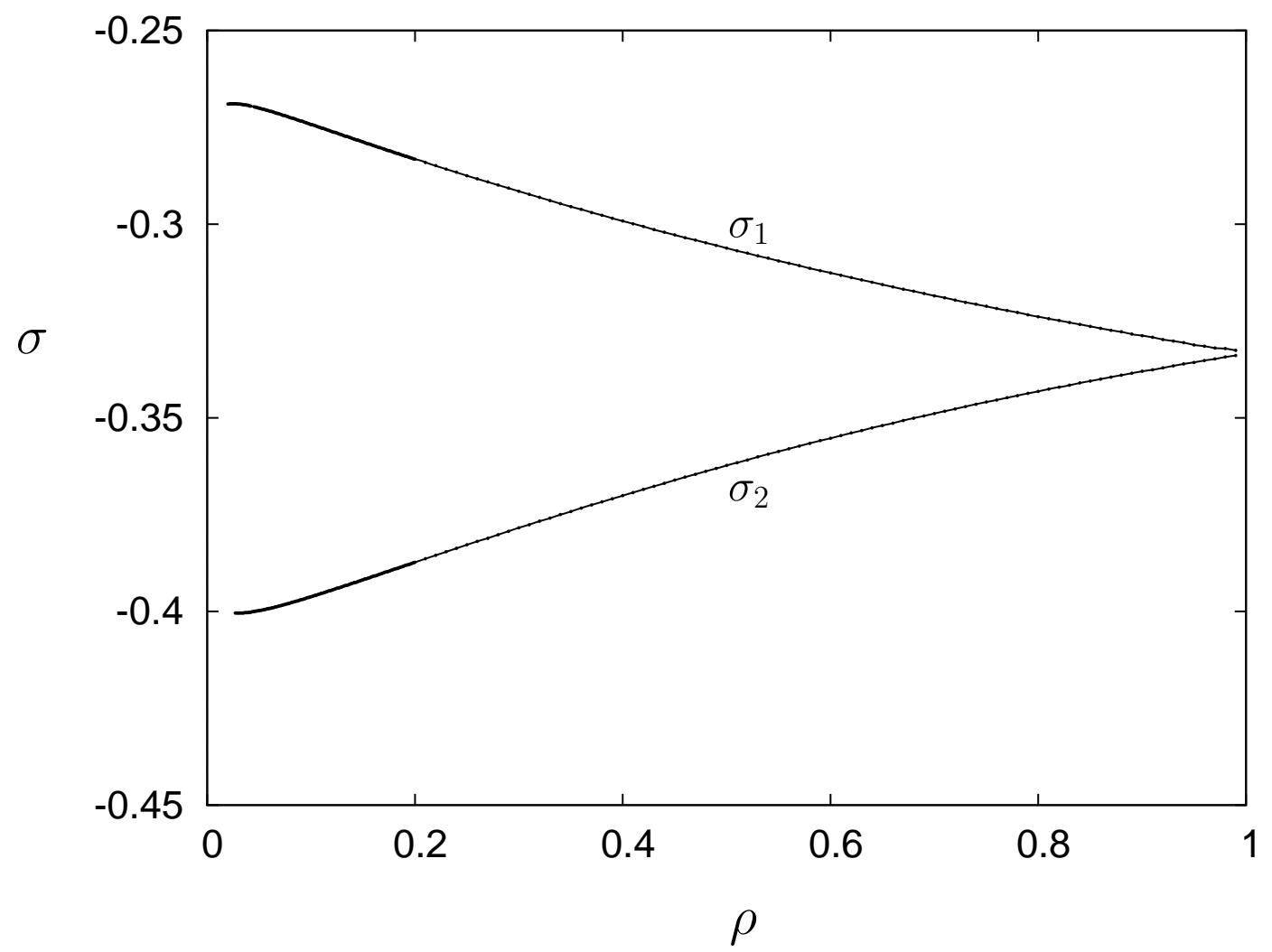

Figure 3. Gap region as a function of $\rho$ for $\nu=1.0$. No similarity solutions exist for values of $(\sigma, \rho)$ between these curves. 

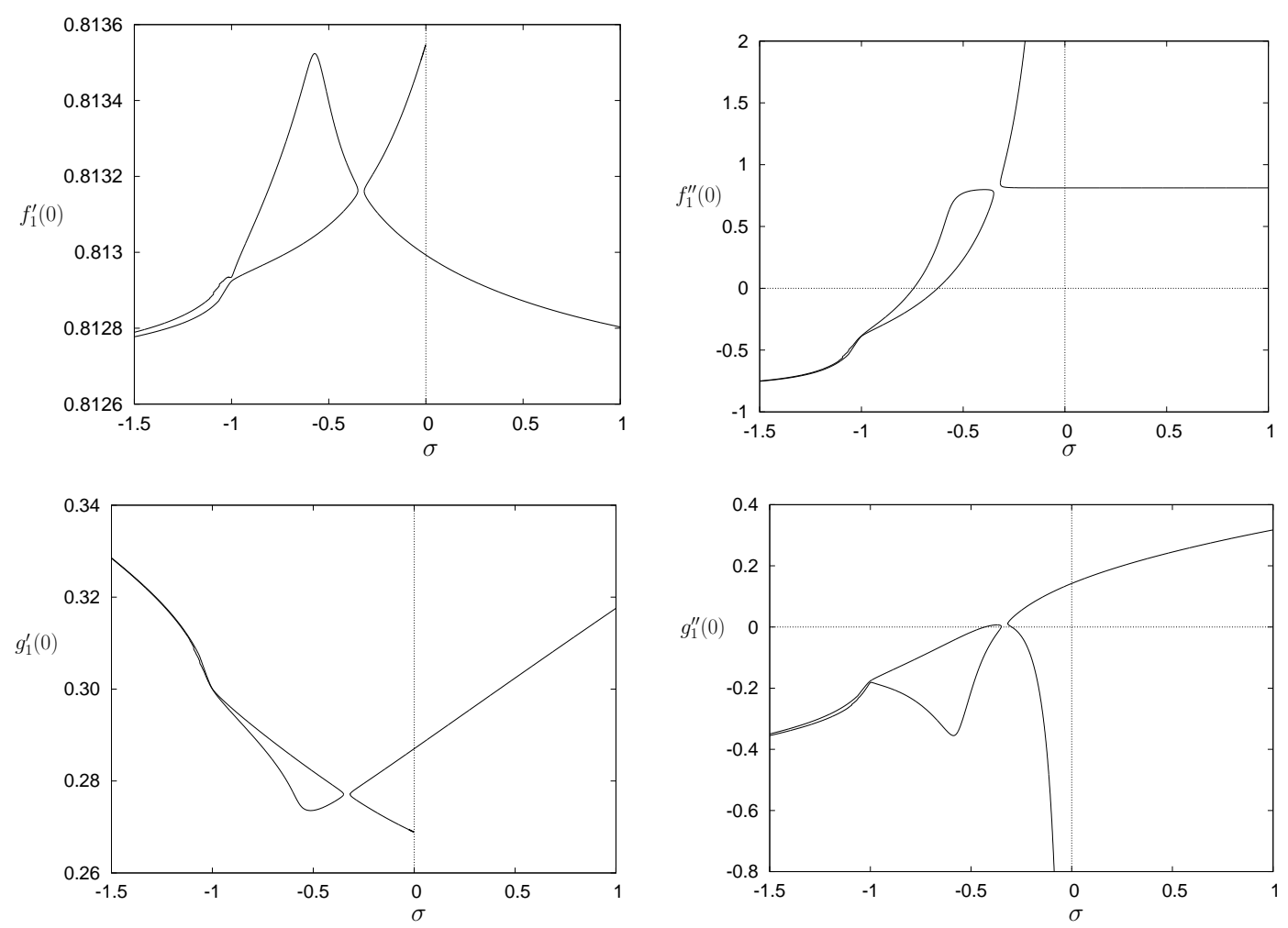

Figure 4a. Interfacial velocities $f_{1}^{\prime}(0), g_{1}^{\prime}(0)$, and upper layer shear stress parameters $f_{1}^{\prime \prime}(0), g_{1}^{\prime \prime}(0)$ for $\rho=0.6$ and $\nu=0.1$. The gap in the solutions lies in the region $\sigma_{2}<\sigma<\sigma_{1}$, where $\sigma_{1}=-0.3174$ and $\sigma_{2}=-0.3498$. 

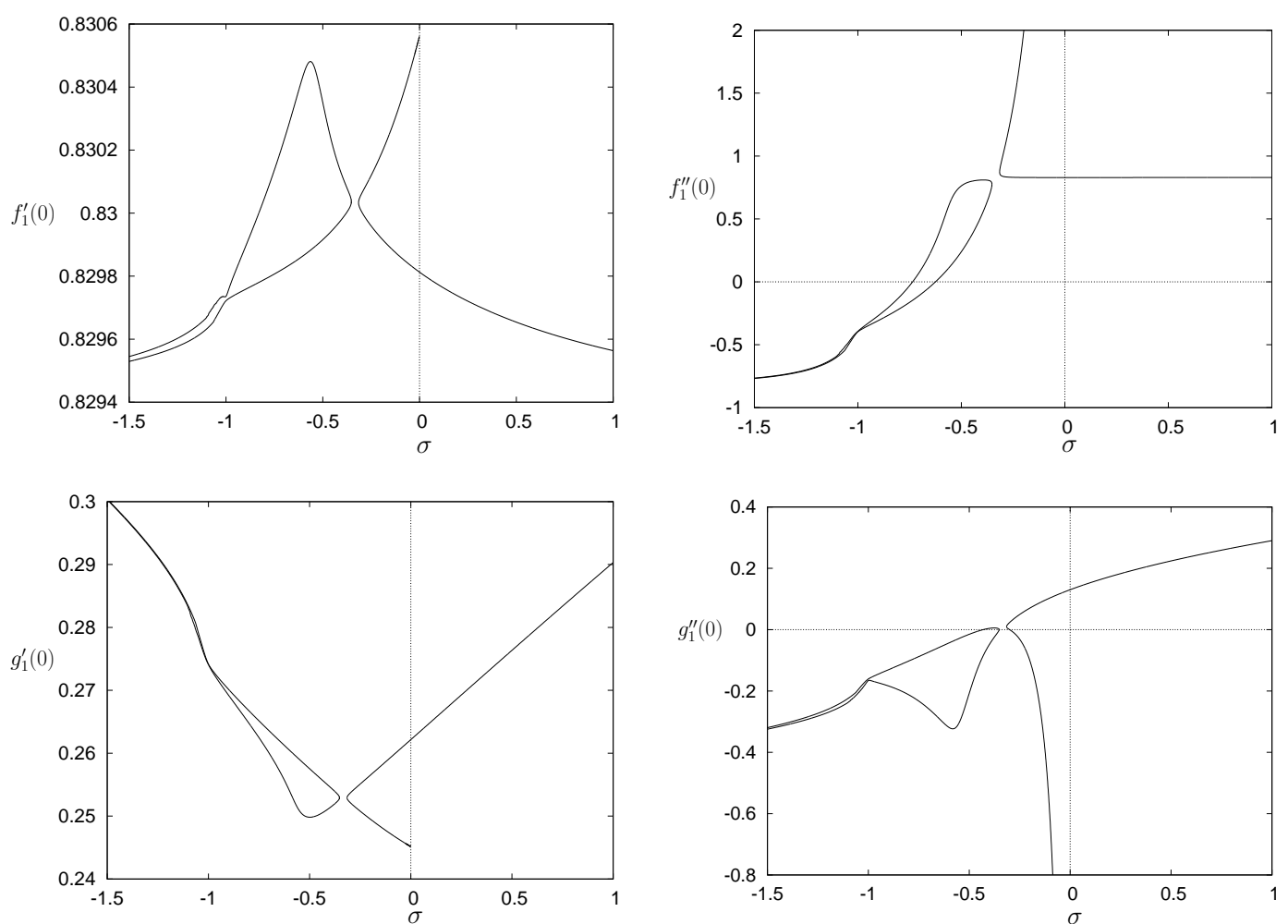

Figure 4 b. Interfacial velocities $f_{1}^{\prime}(0), g_{1}^{\prime}(0)$, and upper layer shear stress parameters $f_{1}^{\prime \prime}(0), g_{1}^{\prime \prime}(0)$ for $\rho=0.6$ and $\nu=0.25$. The gap in the solutions lies in the region $\sigma_{2}<\sigma<\sigma_{1}$, where $\sigma_{1}=-0.3151$ and $\sigma_{2}=-0.3523$. 

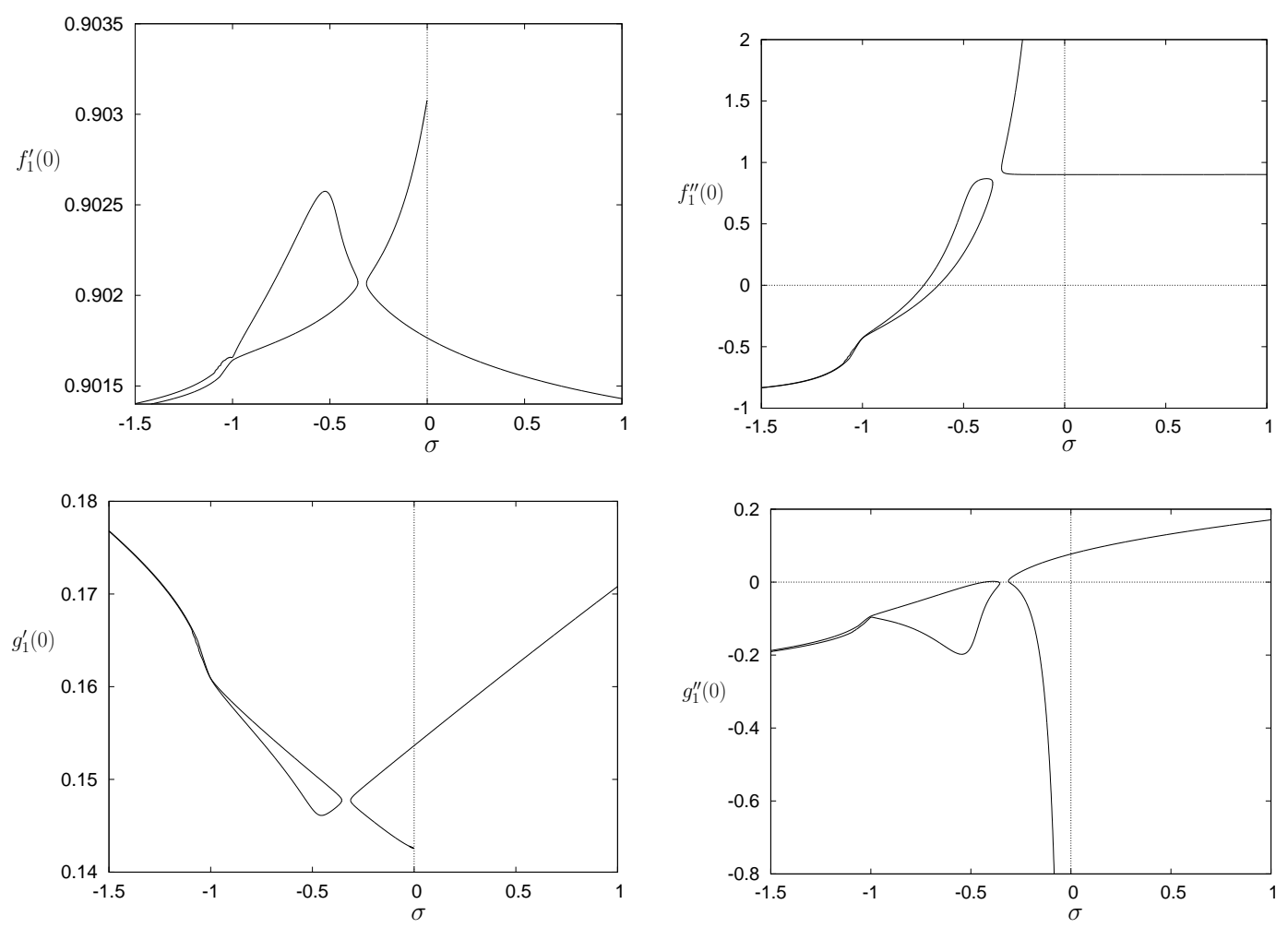

Figure 4c. Interfacial velocities $f_{1}^{\prime}(0), g_{1}^{\prime}(0)$, and upper layer shear stress parameters $f_{1}^{\prime \prime}(0), g_{1}^{\prime \prime}(0)$ for $\rho=0.6$ and $\nu=4.0$. The gap in the solutions lies in the region $\sigma_{2}<\sigma<\sigma_{1}$, where $\sigma_{1}=-0.3121$ and $\sigma_{2}=-0.3558$. 

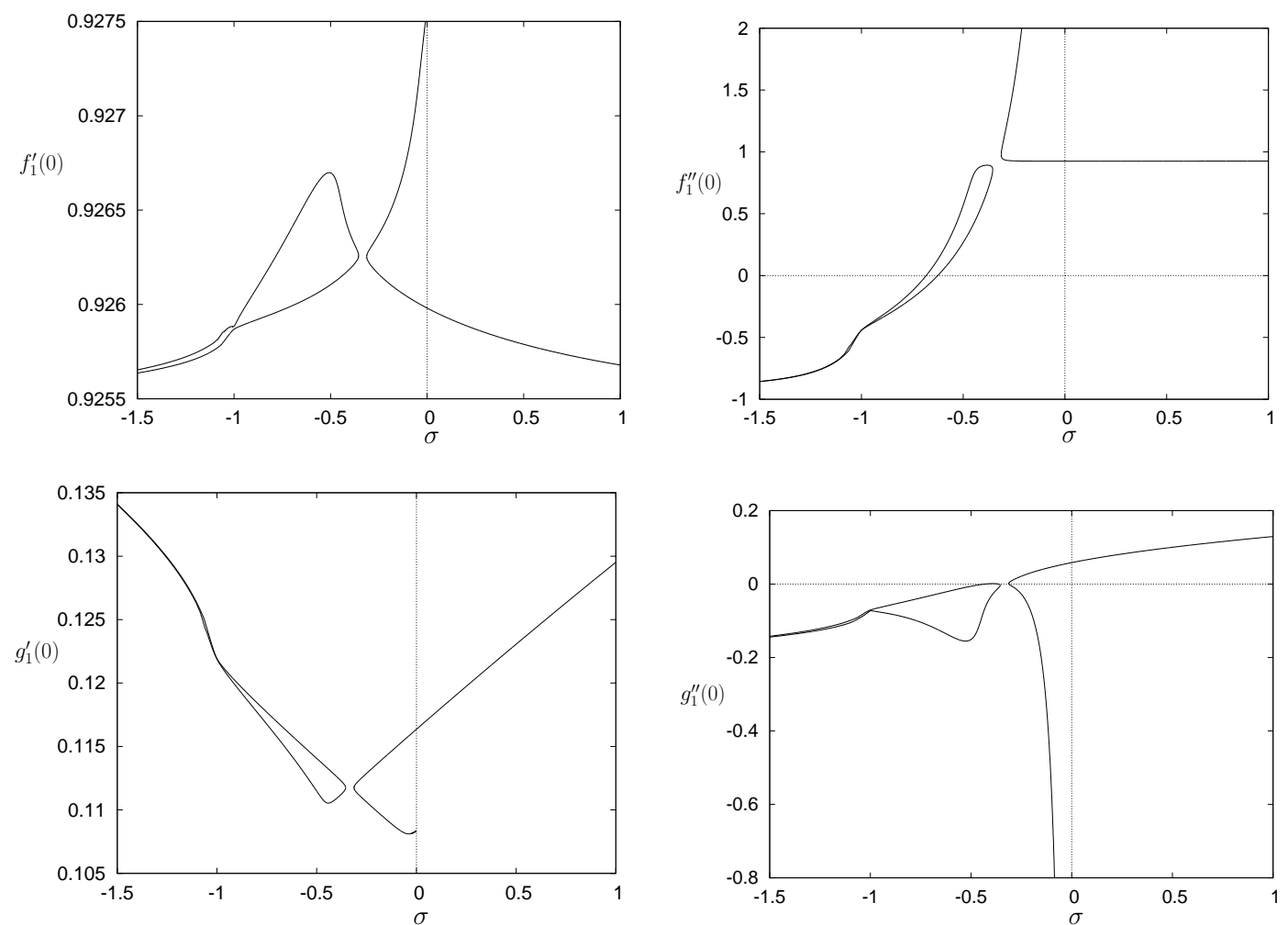

Figure 4d. Interfacial velocities $f_{1}^{\prime}(0), g_{1}^{\prime}(0)$, and upper layer shear stress parameters $f_{1}^{\prime \prime}(0), g_{1}^{\prime \prime}(0)$ for $\rho=0.6$ and $\nu=10.0$. The gap in the solutions lies in the region $\sigma_{2}<\sigma<\sigma_{1}$, where $\sigma_{1}=-0.3130$ and $\sigma_{2}=-0.3548$. 


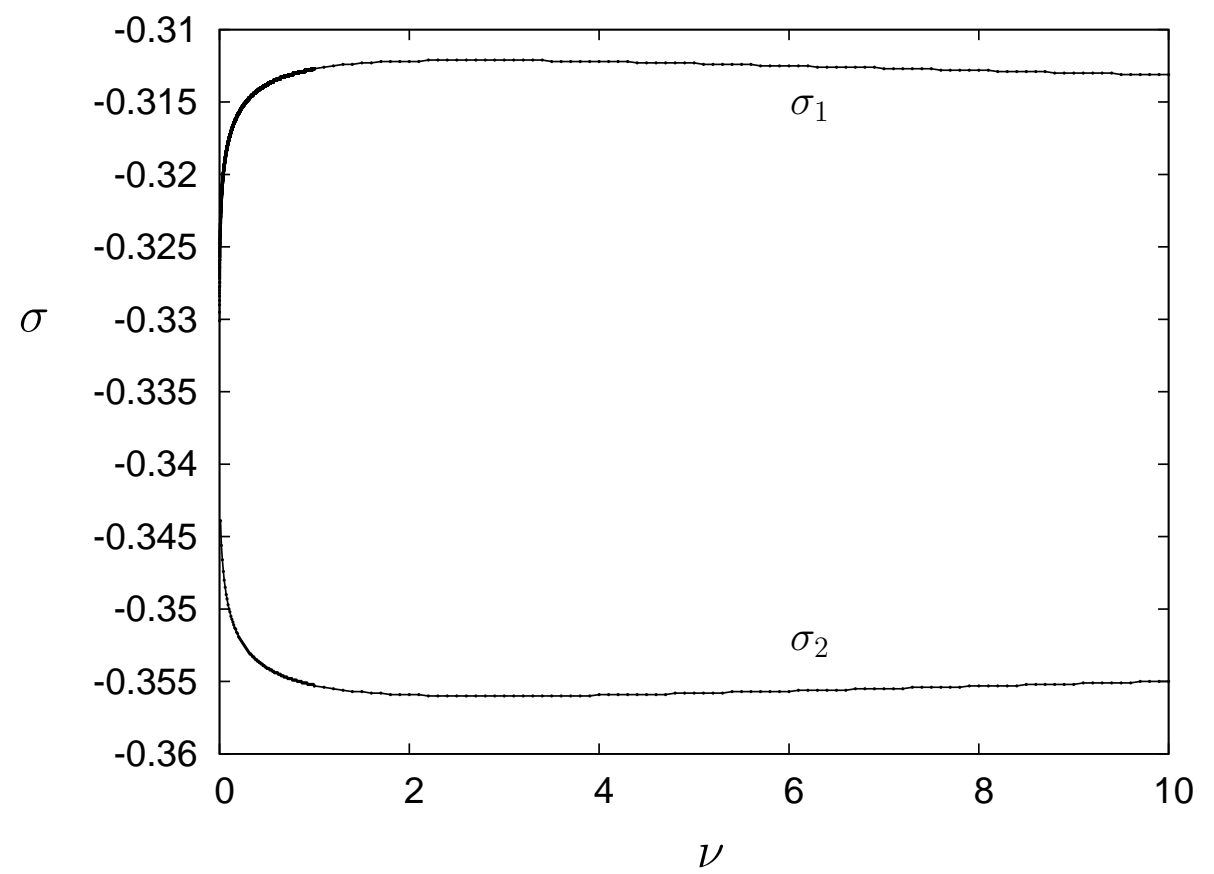

Figure 5. Gap region as a function of $\nu$ for $\rho=0.6$. No similarity solutions exist for values of $(\nu, \sigma)$ between these curves. 


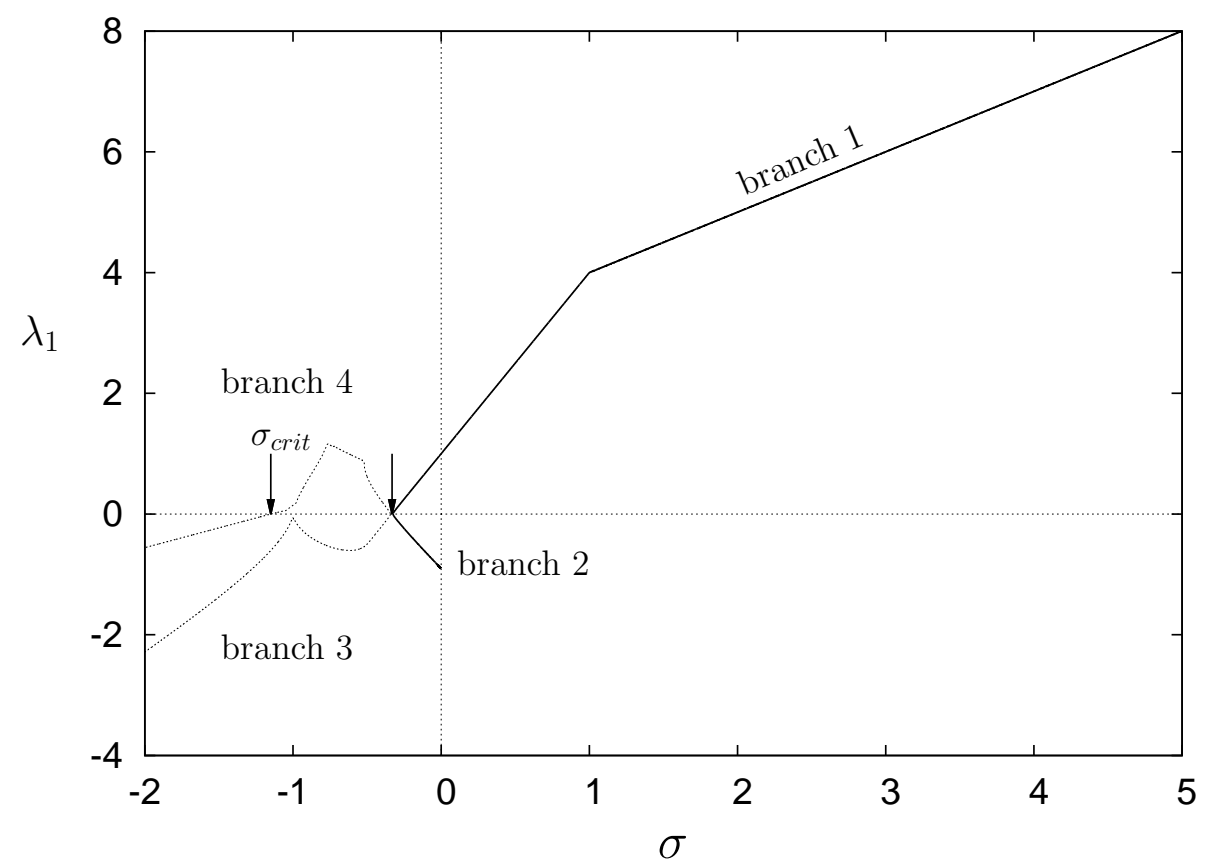

Figure 6. Eigenvalues on the four branches as a function of $\sigma$ for $\rho=0.8$ and $\nu=1.0$. The horizontal arrows show the regions on branch 4 for which the eigenvalues are complex. The downward arrow shows the region of the gap and the upward arrow shows the critical value of $\sigma$ on branch 4 for which the flow changes stability. 


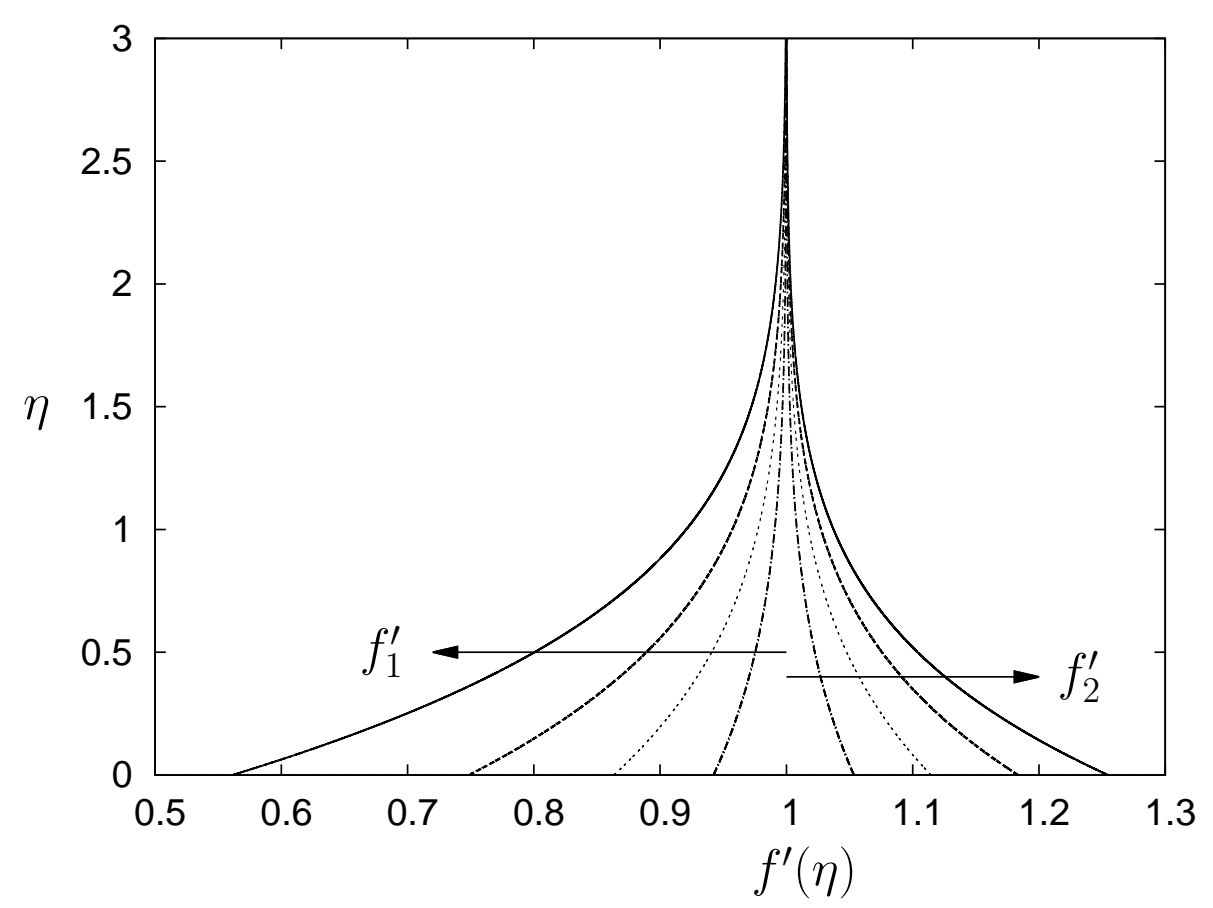

Figure 7a. Velocity profiles $f^{\prime}(\eta)$ for $\sigma=-0.6$ on branch 4 computed at $\rho=$ $\{0.2,0.4,0.6,0.8\}$ with arrows in the direction of decreasing values of $\rho$.

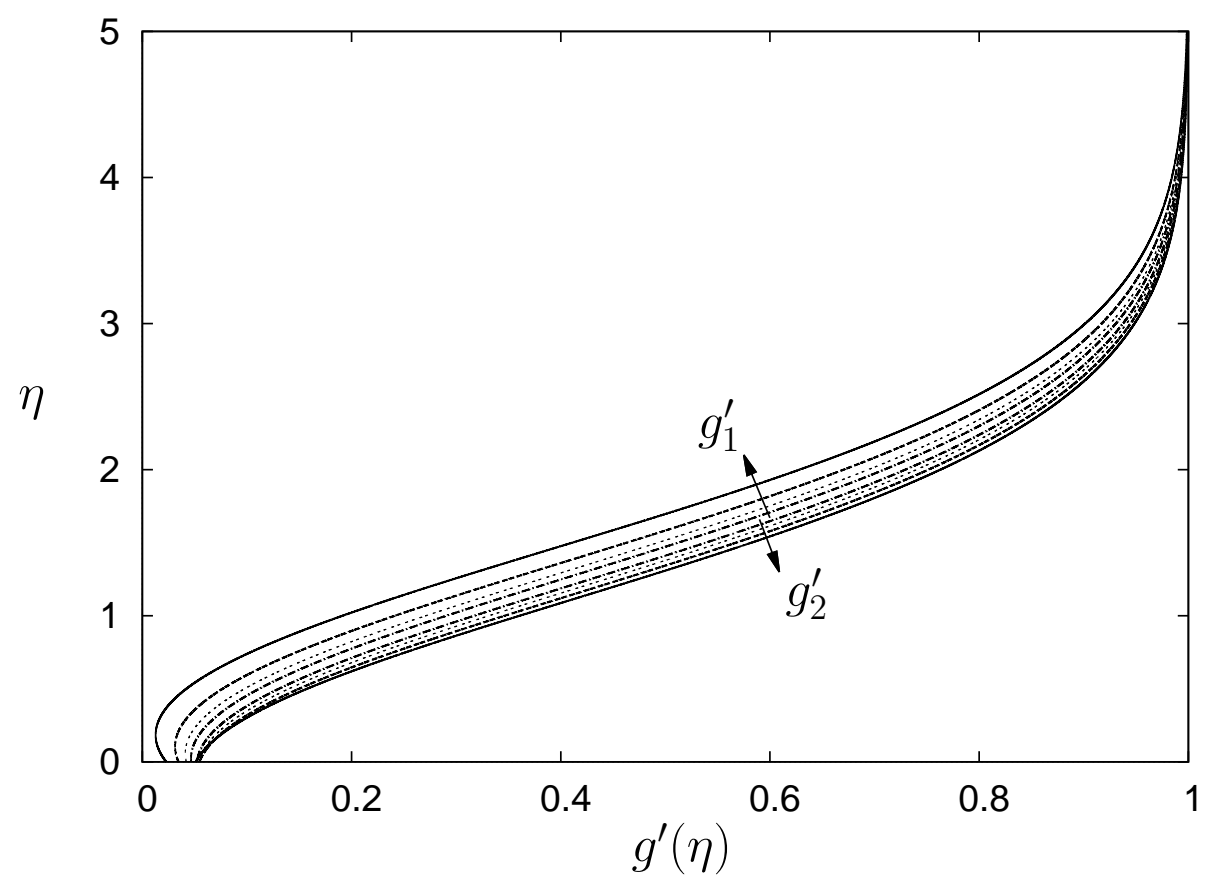

Figure $\mathbf{7 b}$. Velocity profiles $g^{\prime}(\eta)$ for $\sigma=-0.6$ on branch 4 computed at $\rho=$ $\{0.2,0.4,0.6,0.8\}$ with arrows in the direction of decreasing values of $\rho$. 


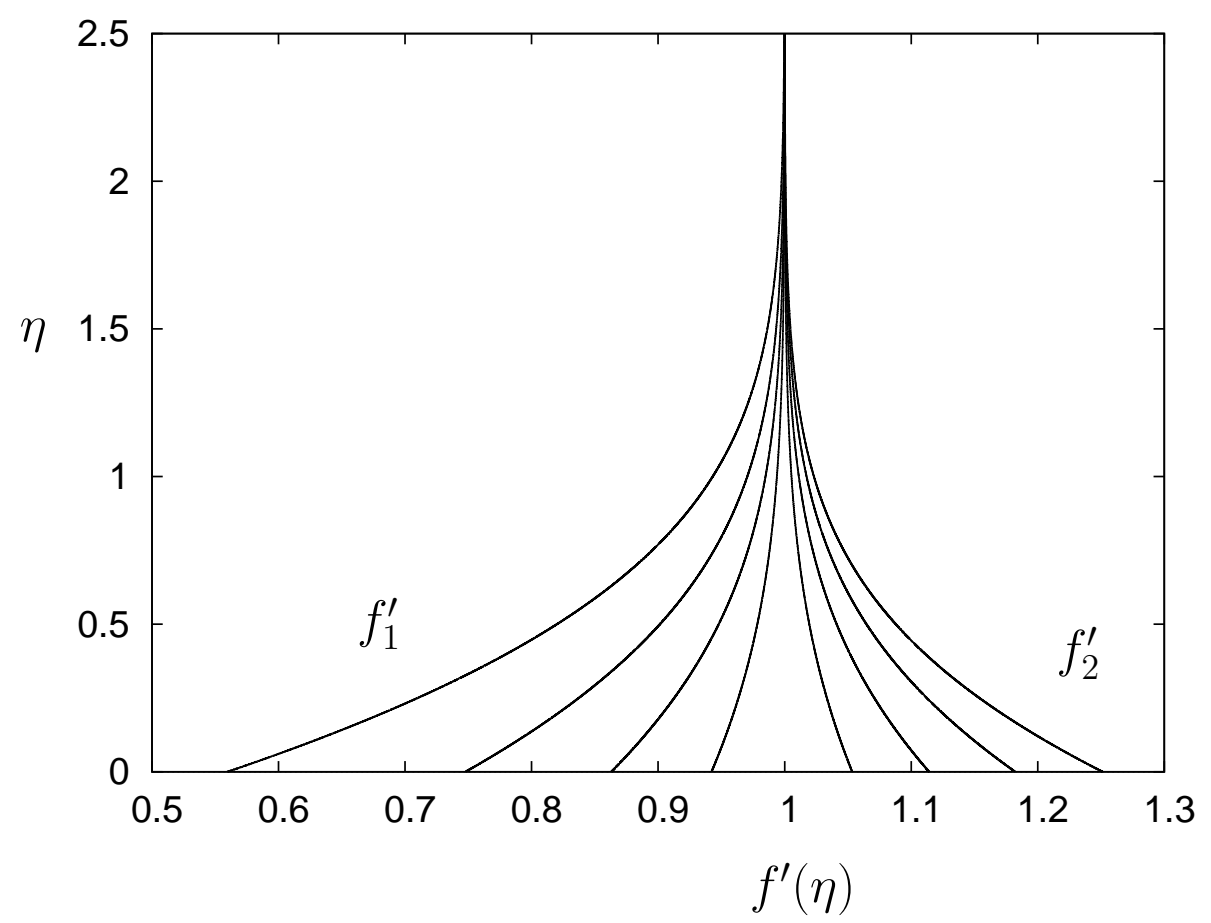

Figure 8a. Velocity profiles $f^{\prime}(\eta)$ for $\sigma=-1.1$ on branch 1 computed at $\rho=$ $\{0.2,0.4,0.6,0.8\}$ with arrows in the direction of decreasing values of $\rho$.

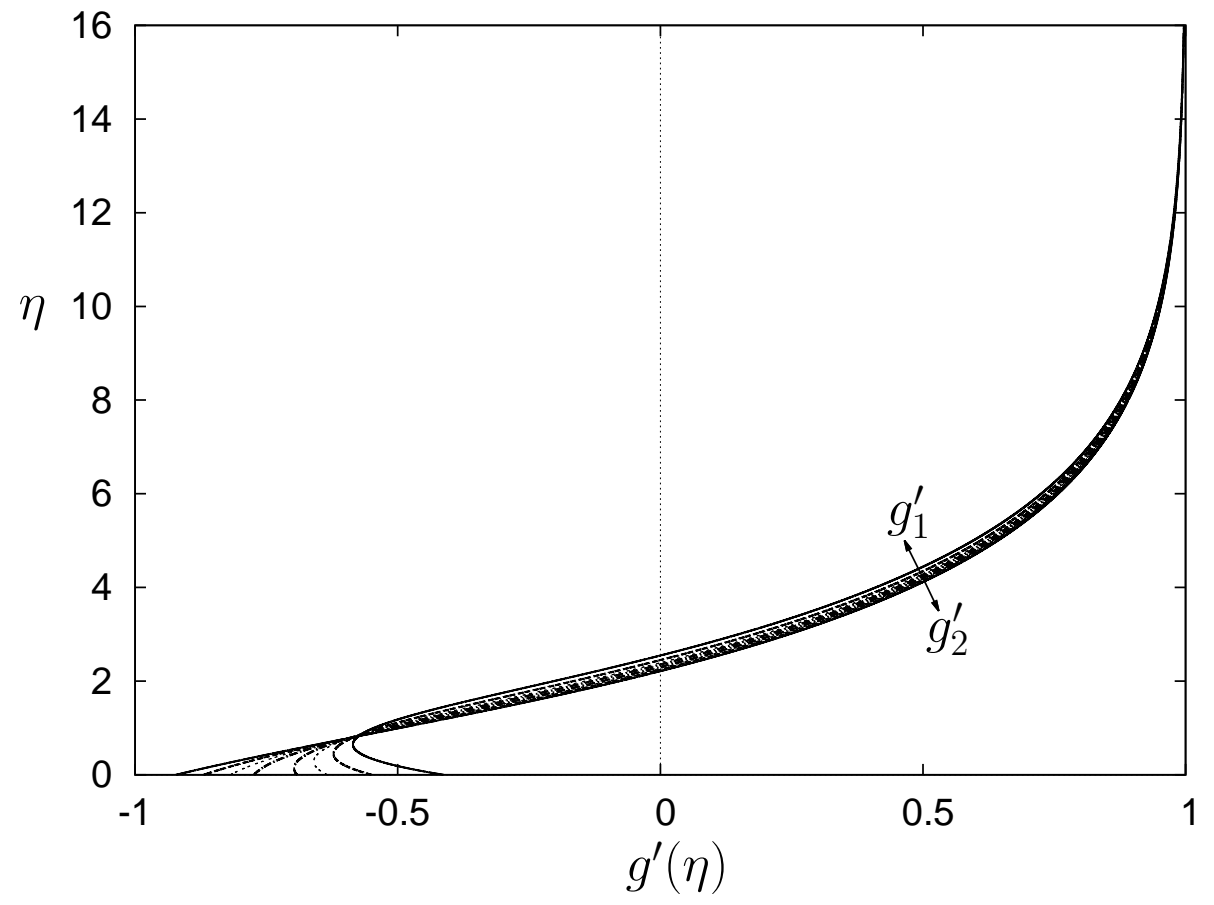

Figure 8b. Velocity profiles $g^{\prime}(\eta)$ for $\sigma=-1.1$ on branch 1 computed at $\rho=$ $\{0.2,0.4,0.6,0.8\}$ with arrows in the direction of decreasing values of $\rho$. 


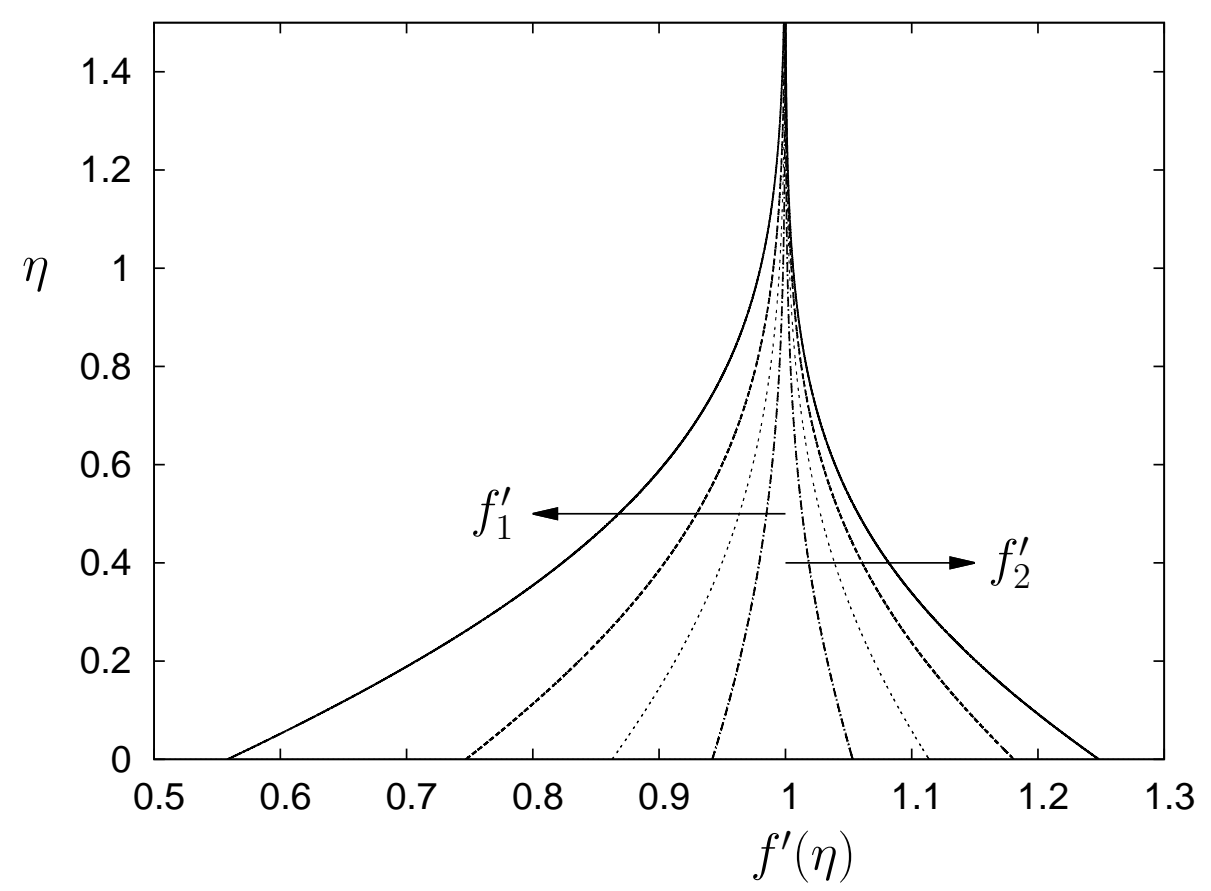

Figure 9a. Velocity profiles $f^{\prime}(\eta)$ for $\sigma=3.0$ on branch 1 computed at $\rho=$ $\{0.2,0.4,0.6,0.8\}$ with arrows in the direction of decreasing values of $\rho$.

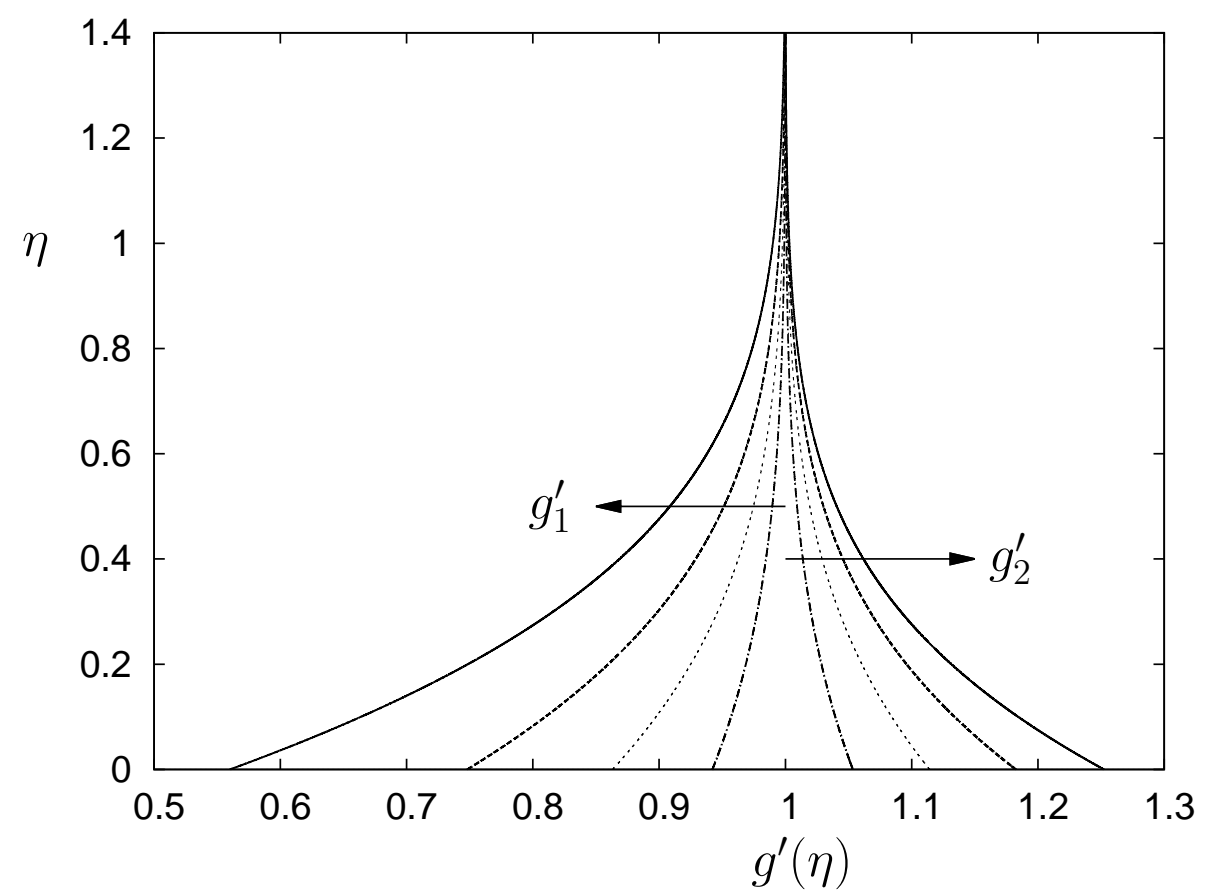

Figure 9b. Velocity profiles $g^{\prime}(\eta)$ for $\sigma=3.0$ on branch 1 computed at $\rho=$ $\{0.2,0.4,0.6,0.8\}$ with arrows in the direction of decreasing values of $\rho$. 